\title{
Resonances of the Earth-Ionosphere Cavity Observed at Cambridge, England ${ }^{1}$
}

\author{
M. J. Rycroft
}

\author{
Cavendish Laboratory, Cambridge, and Space Sciences Division, NASA, Ames Research Center \\ Moffett Field, Calif.
}

(Received February 3, 1965)

\begin{abstract}
A program of observations of the natural vertical electric field in the atmosphere in the band 1 to $30 \mathrm{~Hz}$ is discussed. After rejection of unwanted signals, the ELF (extremely-low-frequency) signal is amplified, its waveform being preserved as a photographic record which can be digitized automatically. The power spectral density of the field is computed, and at all times shows statistically significant peaks at 8 and $14 \mathrm{~Hz}$, with evidence of other peaks at 20 and $26 \mathrm{~Hz}$. These are the resonant frequencies of modes of the electromagnetic field in the earth-ionosphere cavity, excited by radiation from lightning discharges. A comparison is made between such spectra and those observed elsewhere.

The spectra are also compared with those calculated theoretically. The agreement is only fair for a sharply bounded, homogeneous, isotropic ionosphere concentric with the earth, but better for Galejs' model ionosphere with an exponential conductivity versus height profile. From the derived propagation constant, the attenuation rate in the band 7 to $30 \mathrm{~Hz}$ is found to be approximately 0.25 $\mathrm{dB} / \mathrm{Mm}$, i.e., $0.25 \mathrm{~dB} / 1000 \mathrm{~km}$, and the phase velocity $0.77 \mathrm{c}$. The half width of the thunderstorm belt about an equator (with respect to a pole at Cambridge) is found to be $44^{\circ} \pm 12^{\circ}$, in agreement with thunderstorm statistics. Diurnal and seasonal variations of the power in the various modes are interpreted in terms of nodes of the field and the movement of thunderstorm centers. The fine structure of the spectrum is investigated. A typical measured field strength of $0.34 \mathrm{mV} / \mathrm{m}$ in a $1-\mathrm{Hz}$ bandwidth at $8 \mathrm{~Hz}$ is shown to be reasonable.

The energy in the background spectrum, which overwhelms that in the cavity resonance spectrum below $5 \mathrm{~Hz}$, may be due to nearby lightning activity, local electrostatic effects, or extraterrestrial phenomena.
\end{abstract}

"There is music in the spacing of the spheres," Pythagoras.

\section{Introduction}

Schumann [1952] suggested that modes of the electromagnetic field in the dielectric shell between the earth and ionosphere might be excited to resonance by radiation from lightning discharges. His theory indicated that the observed resonant frequencies of the cavity would be

$f_{n}^{\prime}=f_{1}^{\prime} \sqrt{n(n+1) / 2}$, with $f_{1}^{\prime} \prec c / \sqrt{2} \pi R=10.6 \mathrm{~Hz}$,

in which $n$ is an integer ( $n$ being 1 for the fundamental mode), $c$ is the velocity of light, and $R$ is the earth's radius. The first positive evidence for the existence of this resonant cavity was published by Balser and Wagner [1960]. At that time an observational program concerning natural radio noise below $40 \mathrm{~Hz}$ was initiated in Cambridge, England. A full description of the apparatus used, together with a discussion of some preliminary results and a brief review of related

\footnotetext{
1 Some of the results reported in this paper were presented at the Symposium on Ultra Low Frequency Electromagnetic Fields, Boulder, Colo. August 17-20, 1964.
}

investigations, was presented by Rycroft and Wormell [1964a]. The aim of this paper is to present a sample signal spectrum (having demonstrated how this is obtained experimentally), a mean spectrum of some 30 records, together with their interpretation in the light of current theories on earth-ionosphere cavity resonances.

Natural electromagnetic phenomena in the frequency range of interest have been reviewed recently by Kleimenova [1963].

\section{Experiments}

The antenna used to measure the natural vertical electric field consists of an insulated horizontal wire, of radius $0.15 \mathrm{~cm}$ and length $190 \mathrm{~m}$, supported at an average height of $4.5 \mathrm{~m}$ above a meadow some $2 \mathrm{~km}$ from the center of Cambridge. The signal induced is fed to a series of filters that remove extraneous signals due to powerful local transmitters, oscillations of the antenna excited by wind, and the main power supplies. The signal in the band 1 to $30 \mathrm{~Hz}$ is amplified by a low noise, high gain feedback amplifier, the frequency response of which is shown in figure 1 . 


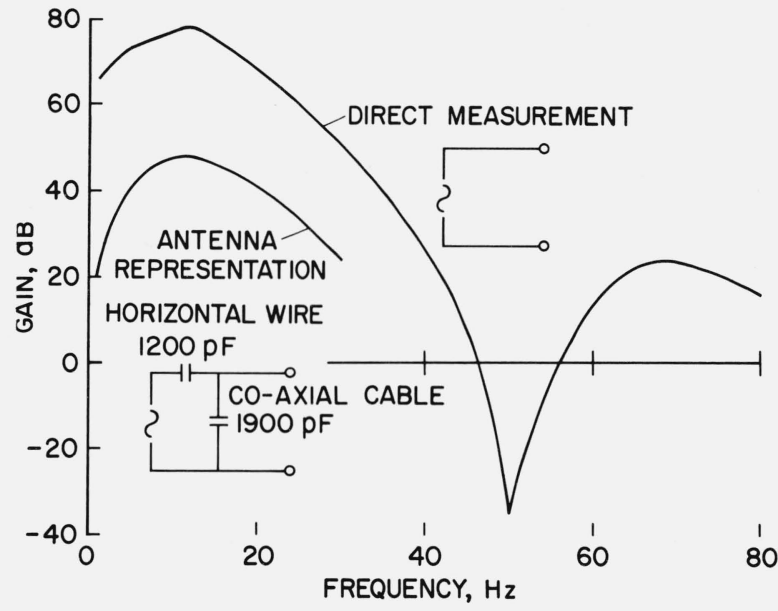

FIGURE 1. Frequency response of the amplifying system. Note that the response measured with a signal generator directly feeding the amplifier input differs considerably from that measured when a representation of the antenna is included.

\section{$21.30 \mathrm{GMT}$ \\ JUNE I, 1962 \\ (a)}

\section{$11.30 \mathrm{GMT}$} JUNE 2, 1962

(b)

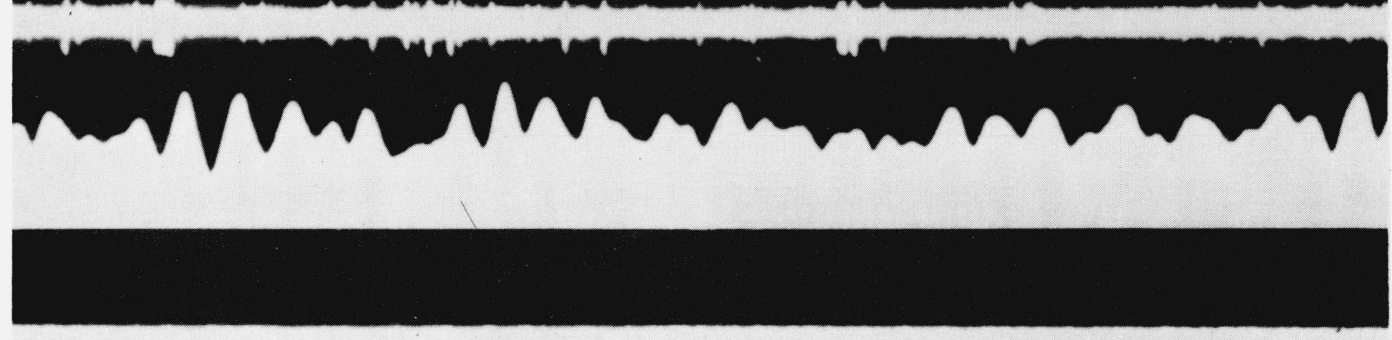

$09.30 \mathrm{GMT}$ JUNE 3,1962
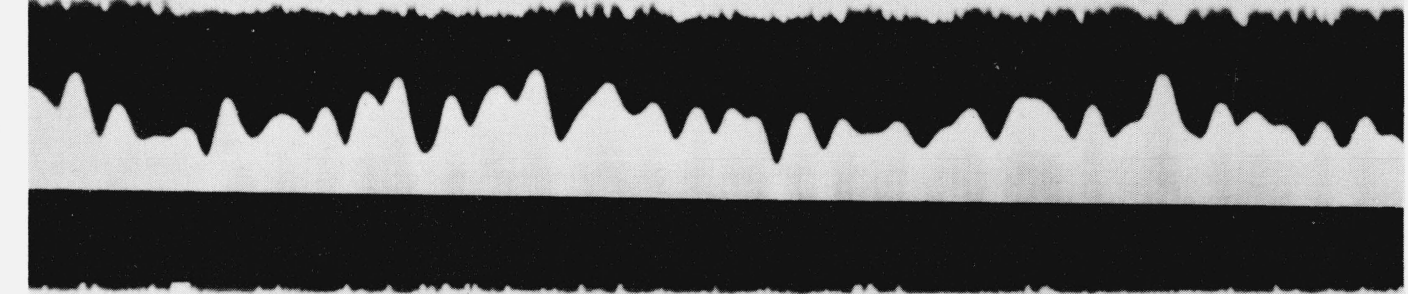

(c)
A comparison of the response obtained when a low impedance signal generator feeds the amplifier (upper curve) with that obtained when a representation of the antenna is used (lower curve) reveals that it is difficult to optimize the coupling between antenna and amplifier. Examples of the extremely-low-frequency (ELF) waveform obtained when an oscilloscope spot is photographed by film moving at $7.06 \mathrm{~cm} / \mathrm{sec}$ are given in figure 2. Above each trace appears the signal in the band 0.5 to $8 \mathrm{kHz}$; only for the strong atmospherics at the left side of figure $2 b$ does the ELF waveform appear correlated with impulses recorded on the kilohertz channel. In general, there is no correlation between individual atmospherics and the ELF trace.

It is evident from figure 2 that there are no predominant frequency components in the signal waveform. In order to search for cavity resonance effects, the amplitude of the ELF trace has to be digitized at intervals of $1.18 \mathrm{~mm}$ (corresponding to $1 / 60 \mathrm{sec}$ ), from which the power spectral density of the record up to $30 \mathrm{~Hz}$ has to be computed. An automatic digitizer, constructed in the laboratory, is used. Its modus operandi is to scan the film record with the well focused image of a spot of light, the scanning

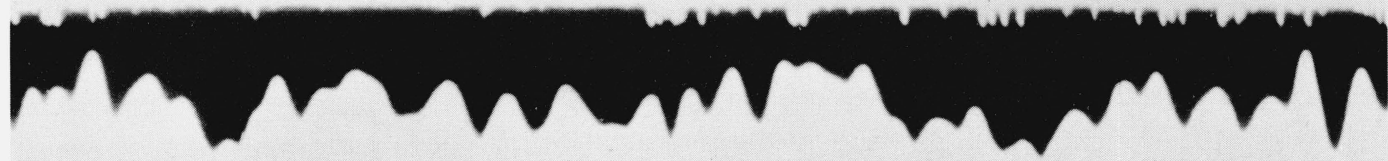

I second

Figure 2. Atmospherics and ELF traces.

Note that in general there is no correlation between individual atmospherics and the amplitude of the ELF waveform. 
mechanism being a rotating plane mirror actuated by a linear cam, cyclically each $3.60 \pm 0.03$ sec. While the spot scans between a mask at the edge of the film and the trace, light shines through the transparent part of the film on to a photomultiplier, during which time pulses of a standard frequency are counted by eight binary counters. When the spot reaches the opaque part of the film, counting is stopped. The count, representing the amplitude of the ELF waveform, is transferred to a tape punch. The counters are reset to zero before the next cycle commences. As the film moves continuously slowly past the scanning region, the amplitude of the radial electric field as a function of time, $E_{r}(t)$, modified by the transfer function of the recording equipment, is obtained on paper tape as desired. Each film is digitized at least twice to eliminate possible eccentricities and malfunctions of the digitizer. Records of different straight lines and sine waves of various frequencies are also digitized. The tapes are translated into the language of the EDSAC computer, either with or without a cubic correction for the nonlinearity of the cam.

\section{Computations}

The power spectral density function of the digitized waveform is computed as the Fourier transform of its autocovariance function [Swinnerton-Dyer, 1963]. A program, developed by Swinnerton-Dyer, removes the mean from the time series, which is then convolved with chosen prewhitening and smoothing functions, and computes the estimated autocorrelation and smoothed power spectral density. The output estimate of the power spectral density is compensated for the prewhitening factor.

It is important to consider the reliability of each estimate. Every record analyzed is at least $T=10$ sec long, composed of $N \geqslant 600$ points at $1 / 60 \mathrm{sec}$ intervals. The power spectral density is required at $(M+1)$ intervals from 0 to $30 \mathrm{~Hz}$; with $M=30$ the spectrum is found at $W=1 \mathrm{~Hz}$ intervals. The number of degrees of freedom of the system is defined as $k=N / M=2 T W$ and is always $\geqslant 20$. Zukerman [1961] has shown that, for $k \geqslant 20$, and 80-percent confidence limits, the estimate of the power spectral density is correct to within $+2.0 \mathrm{~dB}$ or $-1.5 \mathrm{~dB}$. A similar conclusion is reached by Swinnerton-Dyer [1963], who shows that the estimate may plausibly be supposed to behave like $\chi^{2}$ on $k$ degrees of freedom, and by Balser and Wagner [1960], who find the standard deviation of random fluctuations.

The power spectral densities of two series of 600 random numbers generated by the computer are plotted in figure 3 . They are independent of frequency, but have a statistical scatter due to the finite length of the sample of $\leqslant \pm 1.5 \mathrm{~dB}$. A 1.5-dB peak standing out above the background level is therefore statistically significant. The power spectral density of a sample record is plotted in figure 4 , the points being marked by crosses; peaks are evident at $0,8.7,13.1,20.1$, and $26.0 \mathrm{~Hz}$. To test whether these peaks may be spurious ones associated with the d-c peak, a numerical rejection filter at $0 \mathrm{~Hz}$ constructed by the computer is applied to the digitized waveform. The power spectral density of this series is computed and plotted with open circle points in figure 4 . The higher frequency peaks occurring at resonant frequencies of modes of the field in the earth-ionosphere cavity are statistically significant.

From the test waveforms that are digitized, it is concluded that the noise introduced by the digitizer is typically $30 \mathrm{~dB}$ below that in the signal. Power spectral densities computed with and without the correction for the nonlinearity of the cam differ only insignificantly.

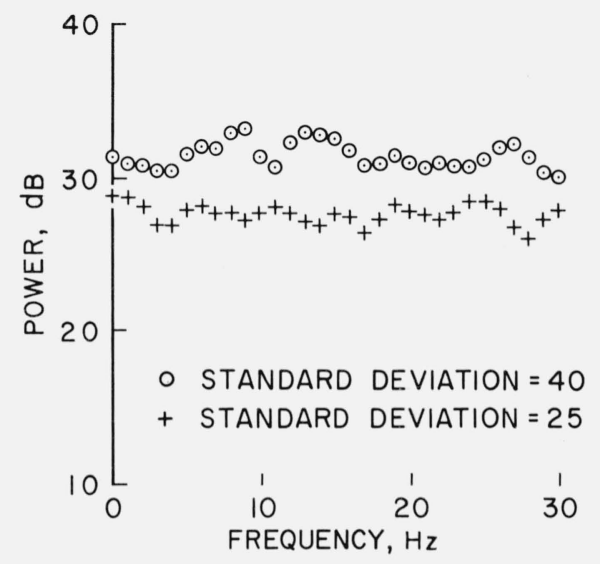

Figure 3. Spectra of two series of 600 random numbers, 0 with a standard deviation of 40 about a mean of 130 , and + with a standard deviation of 25 .

$$
\begin{aligned}
& f_{n}{ }^{\prime}=f_{1}{ }^{\prime} \sqrt{n(n+1) / 2} \\
& f_{1}{ }^{\prime} \simeq 0.75 \mathrm{c} / \sqrt{2} \pi R_{E}
\end{aligned}
$$

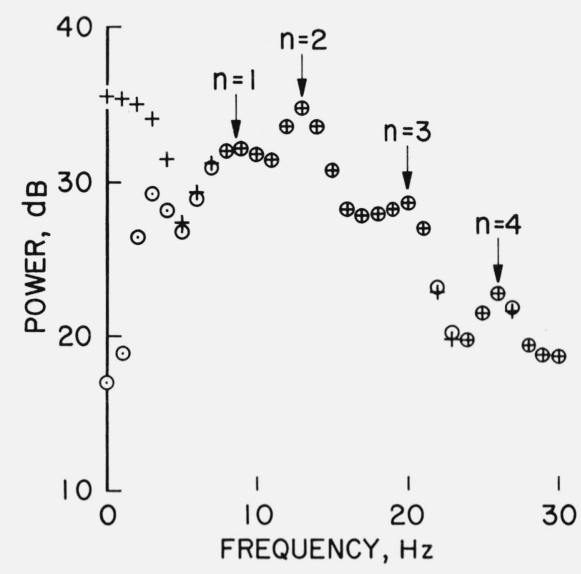

FigurE 4. Spectrum of the digitized signal waveform observed at 16.18 GMT on January 12, 1963.

The crosses show the spectrum as normally computed, and the open circles, that computed with a numerical rejection filter at $0 \mathrm{~Hz}$. Statistically significant peaks are observed at resonant frequencies of various modes $(n=1,2,3$, and 4$)$ of the field in the earth-ionos. phere cavity. 


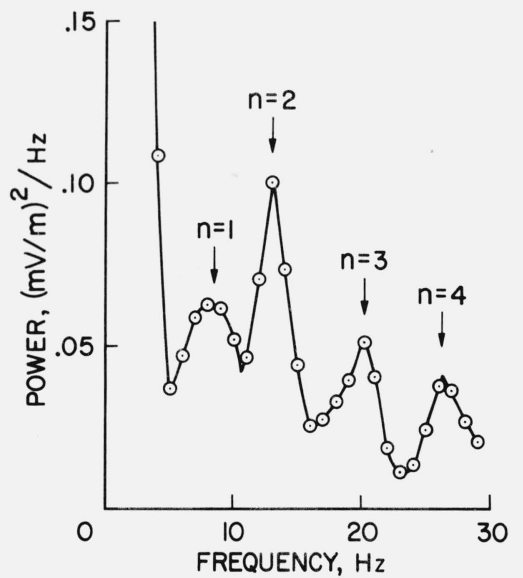

FIgURE 5. Spectrum of the natural vertical electric field observed at 16.18 GMT on January $12,1963$.

This is the spectrum presented in figure 4 corrected for the receiver noise and frequency response. The power ordinate is on a linear, rather than a logarithmic, scale.

\section{Spectra}

The power spectral density of a sample of receiver noise obtained with the amplifier input grounded, which is typically $>20 \mathrm{~dB}$ below that of the ELF signal when new high-tension batteries are used in the amplifier, is subtracted from that of the signal. This spectrum is corrected for the receiver response (fig. 1) and is plotted in figure 5 . The linear power scale emphasizes the peaks in the spectrum more than the logarithmic one. In fact, this second harmonic peak is the sharpest one, having the highest $Q$ value, observed during the experiment. Peaks are noted at $8.5,13.1,20.2$, and $26.2 \mathrm{~Hz}$, which are identified as resonant frequencies of the earth-ionosphere cavity. Spectra are obtained for records taken randomly during meteorologically calm periods in June and December 1962, January, March, May, and July 1963. The first two resonant modes of the earth-ionosphere cavity are clearly shown in all records, together with evidence for the next two. From the best attempts at digitizing 31 records, the mean ELF spectrum is calculated and plotted in figure 6 .

Knowing the amplifier gain, its frequency response, and the electrostatic characteristics of the antenna, the absolute power calibration shown in figures 5 and 6 is derived. This is checked having measured the signal induced by GBR transmissions. At $8 \mathrm{~Hz}$ the mean power spectral density is $0.117(\mathrm{mV} / \mathrm{m})^{2} / \mathrm{Hz}$, with a standard error of the mean of $\pm 0.008(\mathrm{mV} / \mathrm{m})^{2} /$ $\mathrm{Hz}$. This may be expressed as an equivalent amplitude of $0.342 \pm 0.012 \mathrm{mV} / \mathrm{m}$ in a $1-\mathrm{Hz}$ bandwidth at $8 \mathrm{~Hz}$. This value is in agreement with earlier quoted values [Large and Wormell, 1958; Balser and Wagner, 1962; Rycroft and Wormell, 1964a]. It is equivalent to a Poynting flux of the order of $10^{-10} \mathrm{~W} / \mathrm{m}^{2} / \mathrm{Hz}$.

The shapes of the spectra differ from those of sample spectra presented earlier [Rycroft and Wormell, 1964a, (figs. 8, 9, and 10)] because at that time differences between the frequency response shapes (fig. 1) were ignored. The spectra presented here are similar to those of the magnetic field recorded in France by Gendrin and Stefant [1964] and Stefant [1963]. The main difference between these spectra and those presented by Balser and Wagner [1960] from observations made in Massachusetts is that the power spectral density at the minima between peaks is not independent of frequency, but decreases with increasing frequency. This point may be made in another way by noting that only when the background spectrum shown by the dashed line in figure 6 is subtracted from the ELF spectrum do these observations compare with those of Balser and Wagner [1960]. Although some of this background noise is introduced by the averaging process, it is probable that part represents a real noise source. This could be due to comparatively nearby lightning activity, energy propagated along a geomagnetic line of force, or extraterrestrial phenomena.

Below $5 \mathrm{~Hz}$, the relatively immense power spectral density cannot be explained solely by energy from lightning and is ascribed to local electrostatic effects (as mentioned by Rycroft and Wormell [1964a]), fluctuations of the fair-weather field, or distant geoelectromagnetic phenomena. In figure 7 , the power spectral density of the natural vertical electric field in a $1-\mathrm{Hz}$ bandwidth at $1 \mathrm{~Hz}$ is plotted against the fair-weather vertical electric field measured by a field mill at the time of the record. An association between the two quantities is apparent. At a certain value of the fair-weather field, the power at $1 \mathrm{~Hz}$ tends to be larger the greater the wind force. This could be explained by the wind exciting harmonics of the oscillating antenna, or by clouds of space charge drifting past the antenna. Based on their observations of the natural magnetic field, Lokken, Shand, and Wright [1963] have asserted that lightning dominates the spectrum down to 1 or $2 \mathrm{~Hz}$.

A few observations were made with an antenna consisting of three coils, each of 110,000 turns of $0.132-\mathrm{mm}$ diam wire which was enamel coated, mounted on a mu-metal rod $1 \mathrm{in}$. in diameter and $36 \mathrm{in}$. long. The induced signal was amplified and recorded as described in section 2; sample spectra showed the cavity resonance effect. On one occasion, at 21.00.13 GMT on May 14, 1963, the waveform changed in 4 sec from a typical cavity resonance waveform to a large amplitude (off-scale) wave at $8.0 \mathrm{~Hz}$. This may be a manmade signal or an example of the ELF "noise storms" or "emissions" reported by Llanwyn Jones [1963].

Throughout the course of the experiments, the Mie scattering standing wave patterns required by Boyer's [1961] theory for the formation of the Van Allen trapped radiation belts were not detected.

\section{Theoretical Considerations}

In this section a review of the theories concerning earth-ionosphere cavity resonances, indicating their limitations, is attempted. Rationalized MKSA units are used. 


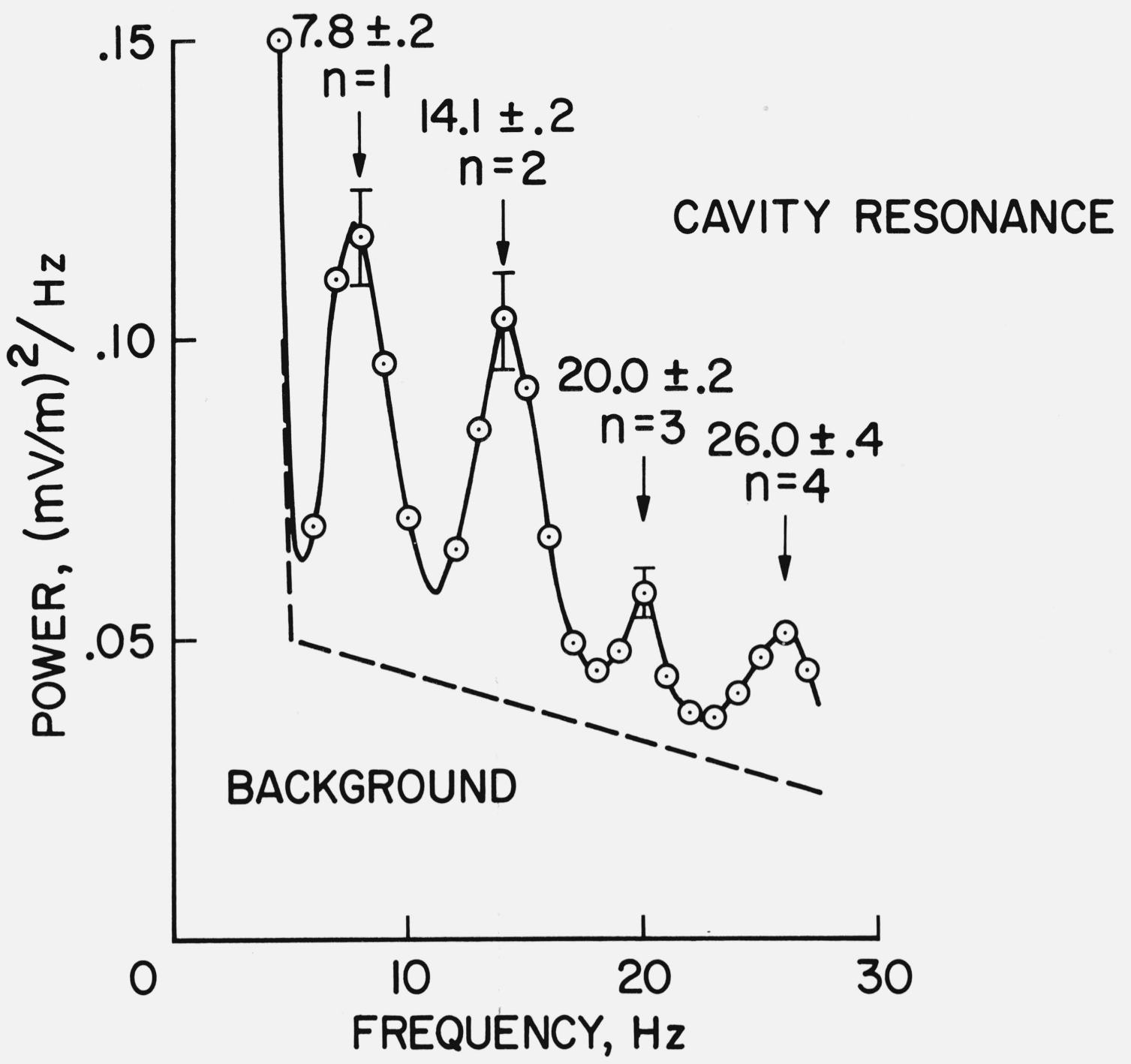

FigURE 6. Mean ELF spectrum of the natural vertical electric field observed in 1962 and 1963.

The similarity of this spectrum to those presented by Gendrin and Stefant [1964] and Stefant [1963] may be noted. Only when the background spectrum shown by the dashed line is subtracted from the mean ELF spectrum do these observations compare with those of Balser and Wagner [1960]. The calculations of Galejs [1963] may be fitted to the observations of Balser and Wagner [1960]. The bars denote the standard error of the mean power at each resonance, derived from 31 records.

Figure 7. Dependence of the power spectral density of the vertical electric field in a $\mathrm{l}-\mathrm{Hz}$ bandwidth at $1 \mathrm{~Hz}$ on the strength of the wind and the magnitude of the fair-weather field.

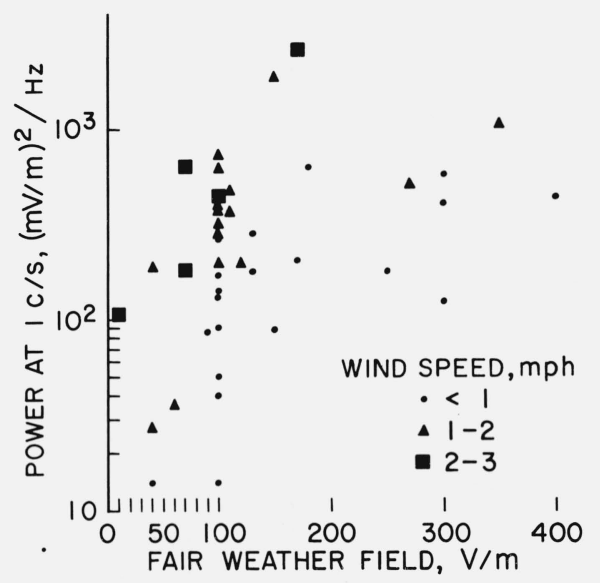


The simplest model, used by Schumann [1952] and Wait [1960], is that of a perfectly conducting earth of radius $R$, surrounded by a thin dielectric shell of height $h, h$ being $\ll R$. This is surrounded by a concentric, sharply bounded, homogeneous, isotropic, good conducting model ionosphere of infinite extent.

A current $I$ flowing vertically in the air near the ground forms, with its image in the ground, an electric dipole of infinitesimal length ds. The dipole moment $\mathbf{M}$ is given by

$$
\frac{\mathbf{d} \mathbf{M}(\omega)}{d t} \exp (i \omega t)=I \mathbf{d s}(\omega) \exp (i \omega t) .
$$

Near a Hertzian dipole, for which $d s \ll \lambda$, the free space wave equation for the Hertz vector must be satisfied. Near the source, where neither the ionosphere nor the earth's curvature is considered, the vertical electric field is given as a sum of electrostatic, induction, and radiation fields [Pierce, 1960]. It is obviously invalid to use the radiation field approximation of this equation in relation to cavity resonances.

A solution of the wave equation in spherical polar coordinates is the transverse magnetic (TM) wave with zero radial magnetic field. For example, in the air layer,

$$
\begin{aligned}
& E_{r}(\omega, t)=-i \frac{\omega}{\sqrt{k^{3} r^{3}}} \\
& \quad \nu(\nu+1) P_{\nu}(\theta)\left[A H_{\nu+1 / 2}^{(1)}(k r)+B H_{\nu+1 / 2}^{(2)}(k r)\right] \exp (i \omega t)
\end{aligned}
$$

in which $k=\omega / c$ is the free space wave number and $\nu+\frac{1}{2}$ is the order of the Hankel function; $\nu$ is determined by the boundary conditions. The DebyeWatson representation of the Hankel function, which is really a W.K.B. approximation to the radial part of the wave equation, is used. The approximations are only valid for $\left|\nu+\frac{1}{2}\right|>1$ and $k r>>1$; the latter inequality is not met at cavity resonance frequencies. Hence Wait [1960] obtains a waveguide mode condition in terms of Fresnel reflection coefficients. The sine of the complex angle of incidence is given by

$$
S=\frac{\nu+\frac{1}{2}}{k R} \simeq \frac{\sqrt{\nu(\nu+1)}}{k R} \simeq 1-\frac{i}{2 k h N_{I}} \simeq 1-\frac{1}{2 h} \sqrt{\frac{i^{3}}{\mu_{0} \omega \sigma_{I}}}
$$

in which $N_{I}$ is the refractive index of the ionosphere, which may be approximated in terms of the conductivity $\sigma_{I}$ above $55 \mathrm{~km}$ (see, for example, Goldberg [1963]). Equation (4) may be rearranged, using $p=i \omega$, to give the modal equation

$$
p^{2}+\frac{1}{h \sqrt{\mu_{0} \sigma_{I}}} p^{3 / 2}+\nu(\nu+1) \frac{c^{2}}{R^{2}} \simeq 0 .
$$

Row [1962] derives the modal equation by another method.
Since only the zero-order waveguide mode is important at cavity resonator wavelengths, the resonator mode equation, of order $n$, is obtained,

$$
\begin{array}{r}
E_{r}(\omega, t)=\frac{I d s(\omega)}{4 \pi h R^{2} \epsilon_{0} \omega} \sum_{n=0}^{\infty} P_{n}(\cos \theta) \frac{\nu(\nu+1)(2 n+1)}{n(n+1)-\nu(\nu+1)} \\
\exp (i \omega t) .
\end{array}
$$

The $n$th resonance of the radial electric field occurs when

$$
\frac{1}{h \omega} \frac{\nu(\nu+1)(2 n+1)}{n(n+1)-\nu(\nu+1)}
$$

is a maximum, $\nu$ being determined by (4) or (5).

For a perfectly conducting ionosphere, the resonant frequencies, $f_{n}$, are determined by (4):

$$
\nu(\nu+1)=n(n+1)=k^{2} R^{2} .
$$

Hence ( 1 ) is derived:

$$
f_{n}=\frac{c}{2 \pi R} \sqrt{n(n+1)} .
$$

For an ionosphere of conductivity $\sigma_{I}$ the resonant frequency is complex; thus the height of the model ionosphere is found to be frequency dependent, which is physically untenable. Equation (5) may be solved under the approximation that

$$
\left|\frac{1}{h \sqrt{\mu_{0} \sigma_{I}}} p^{1 / 2}\right| \ll|p|
$$

as

$$
p \simeq i \omega_{n}^{\prime}-\Omega_{n}
$$

where $\omega_{n}^{\prime}$, the observed resonant frequency, is less than $\omega_{n}$, calculated for a perfectly conducting ionosphere,

$$
\omega_{n}^{\prime}=\omega_{n}\left(1-\frac{1}{h \sqrt{8 \mu_{0} \sigma_{I} \omega_{n}}}\right)
$$

and $\Omega_{n}$ is the amplitude damping coefficient,

$$
\Omega_{n}=\frac{1}{h} \sqrt{\frac{\omega_{n}}{8 \mu_{0} \sigma_{I}}} .
$$

This analysis, used by Gendrin and Stefant [1964] and Rycroft and Wormell [1964a] to explain their observations, leads to a sharply bounded model ionosphere at about $76 \mathrm{~km}$, where the conductivity is $\sim 5 \times 10^{-6} \mathrm{mho} / \mathrm{m}$. It is not strictly valid because the the approximation necessary to derive (8) is not met. The $Q$ values of the resonances calculated from (10) are lower by a factor of 2 than those estimated as the ratio of the observed resonant frequency to the frequency difference at the half-power points. 
Besides fitting experimentally observed values of $f_{n}^{\prime}$ with theoretical ones, the theory based upon a particular model ionosphere must explain the observed $Q$ values, of the order of 4 , which tend to increase with increasing $n$. One more variable is required to describe the ionosphere. Llanwyn Jones [1963] and Chapman and Llanwyn Jones [1964] have achieved excellent agreement for a two-layer model ionosphere. Because the skin depth, $\sqrt{2 / \mu_{0} \sigma \omega}$, is large at these extremely low frequencies, the higher ionosphere regions play a significant part in determining the propagation of ELF electromagnetic energy.

Sharply bounded models do not consider the region where $\sigma / \epsilon_{0} \omega \simeq 1$, typically near $50 \mathrm{~km}$, where displacement currents and conduction currents are of comparable magnitude and where the W.K.B. approximation is not valid. Harris and Tanner [1962] consider this transition region to be as important as the upper region in determining ELF propagation. Galejs [1963] has reviewed his work on a model ionosphere, bounded at a height where $\sigma / \epsilon_{0} \omega=1$, and having an exponential conductivity versus height profile in agreement with observations. Maximizing $E_{r}$ and using the condition that $\operatorname{Re}(S)>>|\operatorname{Im}(S)|$, it is found that (compare (7))

$$
f_{n}^{\prime}=\frac{1}{\operatorname{Re}(S)} \frac{c}{2 \pi R} \sqrt{n(n+1)} .
$$

Wait [1964] obtains a more general form of this equation.

Cosmic ray-induced ionization in the atmosphere contributes to the absorption of ELF radio energy; it is shown [Galejs, 1963] that

$$
\frac{1}{Q}=-2 \operatorname{Re}(S) \operatorname{Im}(S)
$$

TABLE 1. Calculated and observed resonant frequencies of the

\begin{tabular}{|c|c|c|c|c|}
\hline \multirow{2}{*}{ Frequency $(\mathrm{Hz})$} & \multirow[b]{2}{*}{$n=1$} & \multicolumn{2}{|c|}{ Mode order } & \multirow[b]{2}{*}{$n=4$} \\
\hline & & $n=2$ & $n=3$ & \\
\hline $\begin{array}{l}\text { Resonant frequency, } f_{n}, \text { from (7) } \\
\text { Resonant frequency, 0.7642 times } \\
\text { row } 1 \text {; see (11) } \\
\text { Observed } f_{n}^{\prime} \text {, June } 27 \text { and } 28,1960, \\
\bar{B} \text { alser and Wagner [1960] } \\
\text { Observed } f_{n}^{\prime} \text {, mean 1961, 1962, } \\
\text { Chapman and Llanwyn Jones } \\
\text { [1964] } \\
\text { Observed } f_{n}^{\prime} \text {, July } 13 \text { and } 14,1962, \\
\text { Stefant [1963] } \\
\text { Observed } f_{n}^{\prime} \text {, mean spectrum 1962, } \\
\text { 1963, observations at Cambridge, } \\
\text { England }\end{array}$ & $\begin{array}{l}10.60 \\
8.10 \\
7.8 \\
8.0 \pm 0.1 \\
7.85 \pm 0.2\end{array}$ & $\begin{array}{l}18.35 \\
14.03 \\
14.1 \\
14.1 \pm 0.2 \\
14.2 \pm 0.3 \\
14.1 \pm 0.2\end{array}$ & $\begin{array}{l}25.96 \\
19.85 \\
20.3 \\
20.0 \pm 0.4 \\
19.9 \pm 0.3\end{array}$ & $\begin{array}{l}33.52 \\
25.62 \\
26.4 \\
26.0 \pm 0.8 \\
26.25 \pm 0.3\end{array}$ \\
\hline
\end{tabular}
earth-ionosphere cavity

From the mean spectrum presented in figure 6 , the series for $f_{n}^{\prime}$ is found to be $7.8 \pm 0.2,14.1 \pm 0.2,20.0$ \pm 0.2 , and $26.0 \pm 0.4 \mathrm{~Hz}$. These observations are compared with those of other workers in table 1 and with the series 0.7642 times (7). The observed fundamental resonant frequencies are all less than the calculated value in row 2 , but $f_{4}^{\prime}$ is in all cases greater than the calculated value. Hence it is deduced that the $Q$ value tends to increase with increasing $n$. This deduction is confirmed by estimating the $Q$ values from the spectrum.

The real and imaginary parts of $S$ at the four resonant frequencies are thus found. The propagation constant $\gamma$ is related to $S$ :

$$
\gamma=i k S=\alpha+i \Phi
$$

Hence the attenuation constant

$$
\alpha=-k \operatorname{Im}(S) \mathrm{Np} / \mathrm{m}=-0.182 f \operatorname{Im}(S) \mathrm{dB} / \mathrm{Mm}
$$

and the phase velocity is

$$
\frac{\omega}{\Phi}=\frac{c}{\operatorname{Re}(S)}
$$

The attenuation rate is found to be approximately 0.25 $\mathrm{dB} / \mathrm{Mm}$, but is only poorly determined because of the uncertainties in $Q$. The phase velocity is found to vary from $0.75 c$ at $8 \mathrm{~Hz}$ to $0.78 c$ at $30 \mathrm{~Hz}$, with an estimated standard error of $\pm 0.01 \mathrm{c}$.

It is important to consider the effect of the geomagnetic field which makes the ionosphere anisotropic. In the quasi-longitudinal approximation the attenuation is somewhat increased [Wait, 1962]. By considering a radial geomagnetic field and a multilayer stratified ionosphere above $85 \mathrm{~km}$, Thompson [1963] has shown that energy would be lost from the high ionosphere, resulting in $Q$ values decreased from $\sim 16$ to $\sim 8$, especially at night. Madden [private communication, 1964] has extended Thompson's analysis to include the lossy region below $85 \mathrm{~km}$ where displacement currents become important. In the quasitransverse approximation, Wait [1962] and Galejs and Row. [1964] have calculated differences between the attenuation rates for $\mathrm{E}-\mathrm{to}_{\mathrm{O}}-\mathrm{W}$ and $\mathrm{W}-\mathrm{to}_{\mathrm{o}}-\mathrm{E}$ propagation. The effect of -ions on the attenuation constant at cavity

\begin{tabular}{|c|c|c|c|c|}
\hline Material & $\begin{array}{l}\text { Percentage of } \\
\text { earth's surface } \\
\text { covered }\end{array}$ & $\begin{array}{c}\text { Average } \\
\text { depth }\end{array}$ & $\sigma_{E}$ & $\begin{array}{l}\text { Skin depth } \\
\text { at } 8 \mathrm{~Hz}\end{array}$ \\
\hline $\begin{array}{l}\text { Sea water } \\
\text { Basalt, ocean floor }\end{array}$ & $\begin{array}{l}\% \\
71\end{array}$ & $\begin{array}{l}\quad \mathrm{km} \\
0-3 \\
3-12\end{array}$ & $\begin{array}{c}\text { Mho/m } \\
5 \\
10^{-6}\end{array}$ & $\begin{array}{l}80 \mathrm{~m} \\
\text { No effect }\end{array}$ \\
\hline $\begin{array}{l}\text { Rocks } \\
\text { Mantle [Lahiri and } \\
\text { Price, 1939] } \\
\text { Snow, ice }\end{array}$ & $\begin{array}{r}24 \\
5\end{array}$ & $\begin{array}{c}0-35 \\
\\
35-2900 \\
0-1.5\end{array}$ & $\begin{array}{c}10^{-2} \text { to } 10^{-6} \\
10^{-5} \text { to } 10^{-6} \\
10^{-6}\end{array}$ & $\begin{array}{l}2 \text { to } 200 \mathrm{~km} \\
\sim 100 \mathrm{~km} \\
200 \mathrm{~km}\end{array}$ \\
\hline
\end{tabular}
resonance frequencies has been considered by Galejs [1963] and found to be small.

TABLE 2. Electrical properties of materials forming the earth's surface

It has been assumed that the resonances are excited by vertical lightning discharges. The ratio of the far zone radial electric field excited by a vertical dipole to that excited by a horizontal dipole of the same moment (representing an intracloud or intercloud lightning flash) is $>10^{5} \sqrt{\sigma_{E}}$ [W ait, 1960]. Typical values for the conductivity of the earth $\sigma_{E}$ are shown in table 2, drawn from the paper of Watt, Mathews, 
and Maxwell [1963]. The effect of horizontal flashes is thus negligible at extremely low frequencies.

It may also be concluded from table 2 that little ELF energy is absorbed by the earth's surface and therefore that the $Q$ values of the earth-ionosphere cavity are determined entirely by losses in the atmosphere and ionosphere.

\section{Discussion}

The detailed shape of ELF spectra may now be considered, bearing in mind the limitations in the theory mentioned in the previous section.

Equation (6) shows that the spectrum of the radial electric field depends on the spectrum of the exciting lightning discharge. Based on the statistics gathered by Williams [1959], Galejs [1963] has assumed that the return stroke excites the resonances. Pierce [1963] has contended that other processes in a lightning discharge contribute to the ELF noise. These ideas have been extended by Rycroft and Wormell [1964b] who consider the spectrum generated by a "mean" lightning discharge. Accounting for the preponderance of cloud discharges, the result that the spectrum differs little from that of a median return stroke is rather surprising. The spectra computed by Galejs [1963] may thus be used with confidence.

The geographical distribution of sources needs to be considered. Galejs [1961, 1963] has calculated spectra for a uniform distribution of sources over the polar angle $\theta$ in the interval $90^{\circ}-\triangle \leqslant \theta \leqslant 90^{\circ}+\triangle, \theta$ being zero at the receiver. When the background spectrum shown in figure 6 is subtracted from the observed spectrum the resulting cavity resonance spectrum is in good agreement with computed spectra. The ratio of the power at the fundamental $(n=1)$ frequency to that at the second and third harmonics is taken from Galejs [1961] and plotted in figure 8 . The experimental values of Balser and Wagner [1960] are plotted, as are the Cambridge observations, both with and without the background spectrum removed. The observations fit for a half width of the thunderstorm belt about an equator with respect to a pole at Cambridge $\triangle$, of $44^{\circ} \pm 12^{\circ}$. This value is in agreement with that derived from the yearly average geographical distribution of thunderstorms as may be seen from figure 9. This map presents the world in an azimuthal equidistant projection centered on Cambridge; a radius vector is a great circle path on this projection. Also shown are loci of constant $\theta$, for $\theta=90^{\circ}$ where $P_{1}(\cos \theta)=\cos \theta=0$, and for $\theta=55^{\circ}$ and $125^{\circ}$ where $P_{2}(\cos \theta)=\left(3 \cos ^{2} \theta-1\right) / 2 \simeq 0$. According to (6), the radial electric field is zero at these values of $\theta$, for the fundamental and secondorder resonator modes, respectively. Even if a source is present, its effect is not noted by a receiver situated at a certain angle $\theta$ with respect to the source.

It is well known that thunderstorms over tropical land masses occur preferentially during the local afternoon. These two factors which determine the intensity of the cavity resonance signals, termed
CAMBRIDGE :

$\times$ E.L.F. SPECTRUM

- CAVITY RESONANCE -

BACKGROUND SPECTRUM

MASSACHUSETTS:

O BALSER AND

WAGNER, 1960

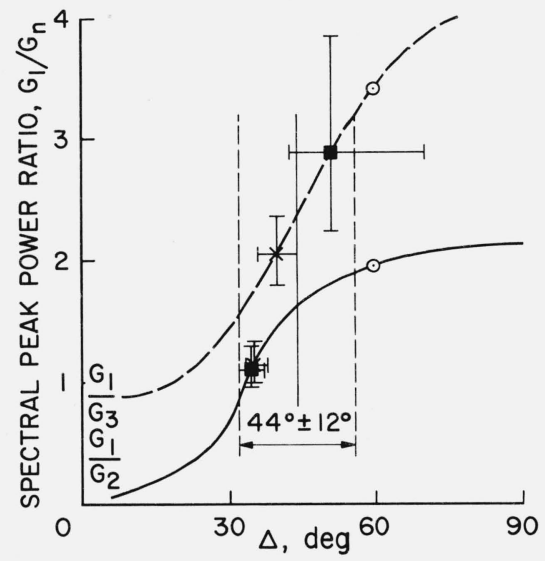

FiguRE 8. Ratio of the power, $\mathrm{G}_{1}$, in the fundamental $(\mathrm{n}=1)$ mode to that in the higher harmonic $(\mathrm{n}=2$ and 3) modes as a function of $\Delta$, the half width of the equatorial thunderstorm belt.

The solid curve shows $G_{1} / G_{2}$, and the dashed curve $G_{1} / G_{3}$, as computed by Galejs [1961]. The open circle points show these ratios observed by Balser and Wagner [1960]. For the Cambridge observations the crosses mark the ratios derived from the mean ELF spectrum; the squares show the ratios derived from the cavity resonance spectrum, the spectrum obtained by subtracting the background spectrum from the mean ELF spectrum. The effect of the error bars in figure 6 is also shown.

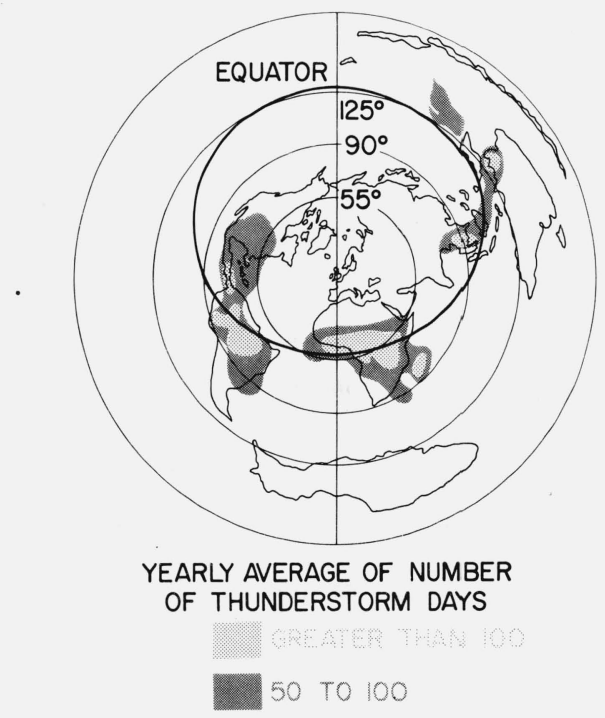

FIGURE 9. Map of the world in an azimuthal equidistant projection centered on Cambridge, England.

The areas of greatest thunderstorm activity averaged over a year are shown [Handbook of Geophysics, 1960]. Loci of constant $\theta$, circles centered on Cambridge, corresponding to minima of the radial electric field in the fundamental and second-order modes, are also shown. 
"mode zeros" and "storm maxima," respectively, were discovered by Balser and Wagner [1962]. A specific example of the effect of these factors is shown in figure 4 ; presumably at this time thunderstorms were active over Brazil. The diurnal variation of the power spectral density in the fundamental mode, presented in figure 10 , is also explained by these two factors. It should be noted that the $n=1$ mode zero at $\theta=90^{\circ}$ masks the Southeast Asia storm maximum effect.

The broad features of the ELF signal diurnal variation, that is the afternoon (GMT) maximum and the nighttime (GMT) minimum, have been noted by Holzer and Deal [1956] (see their fig. 1) and Rycroft and Wormell [1964a] from records similar to their figure 7 . The nighttime minimum is explained by the paucity of thunderstorms over the Pacific Ocean. The solid curve in figure 10 shows a fifth-degree polynomial regression fitted to the points, with a dominant quadratic term. Although there is considerable deviation about the regression, the curve is significant at the 95-percent level. A search was made for seasonal variations in the spectra. The only noticeable feature is that the fundamental peak is relatively weaker than the higher harmonic peaks in winter. The explanation is that the thunderstorm centers over South America and Africa move to the South in winter.

A search was also made for diurnal variations in the resonant frequencies themselves. Complex variations have been reported by Balser and Wagner [1962] and Llanwyn Jones [1963]. The observations reported here are too few and too scattered to allow comparison with previous work. The mean resonant frequencies are $8.0 \pm 0.1,14.0 \pm 0.2,19.8 \pm 0.2$, and $26.1 \pm 0.3$ $\mathrm{Hz}$, which differ slightly from those deduced from the mean spectrum in section 5 .

Neither the power in the fundamental mode nor the resonant frequencies of the first two modes appeared to be correlated with the magnetic $K_{p}$ index at the time of the record.

The power spectral densities of the longest signal records were computed from 0 to $30 \mathrm{~Hz}$ at $0.2 \mathrm{~Hz}$ intervals in order to investigate the fine structure of the cavity resonance spectrum. One such spectrum is shown in figure 11 and may be compared with those presented by Madden et al., [1962] and Chapman and Llanwyn Jones [1964]. These spectra are all extremely jagged. One interpretation is that the radial ELF electric field given by (3) should contain an azimuthal dependence, in which case it would be proportional to

$$
\sum_{m=-n}^{n} P_{n}^{m}(\cos \theta) \exp (i m \varphi) \text {. }
$$

Since the $n$th order mode is not observed to split into precisely $m=2 n+1$ peaks, it must be concluded that the "fine structure" is spurious, being brought about by statistical fluctuations due to the finite length of the sample.

As a final piece of evidence that the observed spectrum is the result of excitation of the earth-ionosphere cavity by lightning discharges, an order-of-magnitude calculation is performed to show that the observed

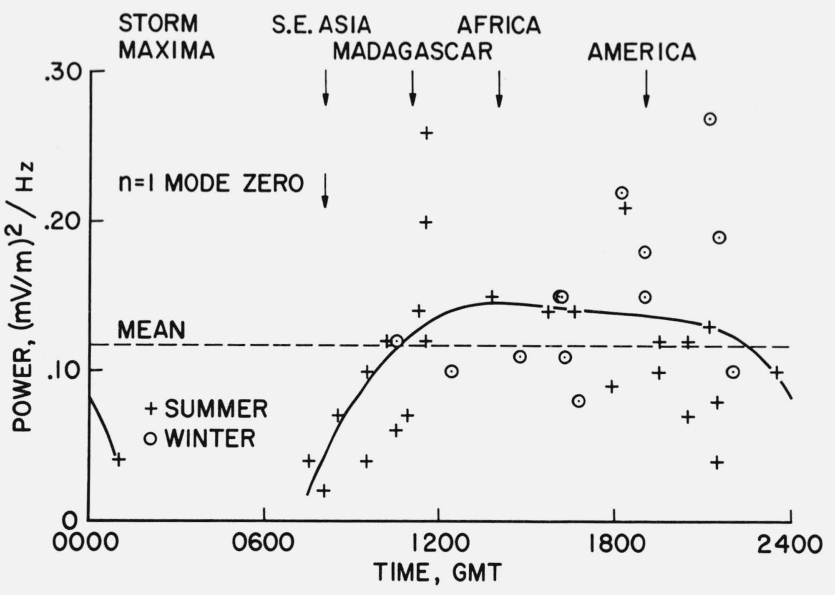

FIgURE 10. Diurnal variation of the power in the fundamental $(\mathrm{n}=1)$ mode.

The crosses are for summer observations and the open circle points for winter ones.

Both "mode zero" and "storm maxima" effects may be noted. The solid curve, a fifthdegree polynomial regression, shows the trend of the diurnal variation.

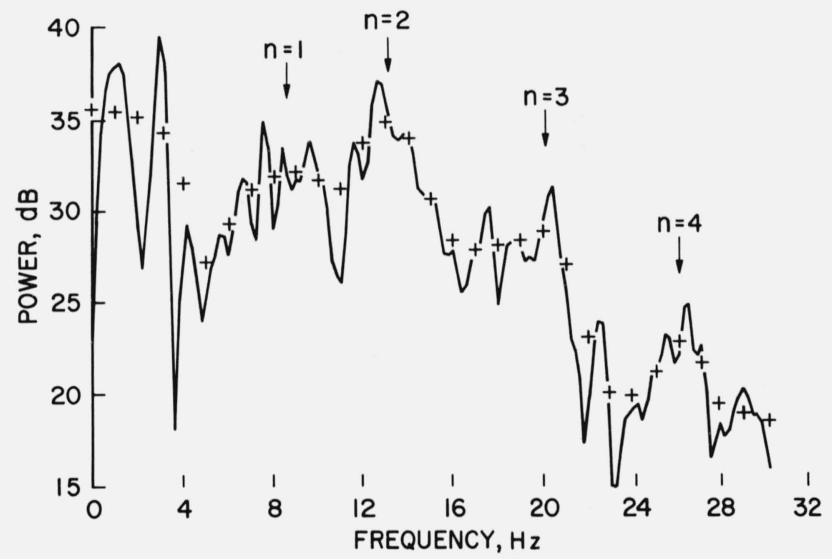

FIgURE 11. Spectrum of the digitized signal waveform observed at 16.18 GMT on January 12, 1963, computed at $0.2-\mathrm{Hz}$ intervals (solid line).

The crosses show the spectrum as normally computed, at $1-\mathrm{Hz}$ intervals, from figure 4 The "fine structure" is not statistically significant.

field strength is reasonable. Inserting $n=1$ for the fundamental resonance, (6) becomes

$$
E_{r}(8 \mathrm{~Hz})=\frac{I d s(8 \mathrm{~Hz})}{64 \pi^{2} h R^{2} \epsilon_{0}} P_{1}(\cos \theta) \frac{3 \nu(\nu+1)}{2-\nu(\nu+1)} .
$$

For one "mean" lightning discharge, Ids $(8 \mathrm{~Hz})$ $\simeq 1.4 \times 10^{5}$ MKSA units [Rycroft and Wormell, 1964b]. It is important to know how many discharges play a part in determining the value of the field at any instant. To estimate this, an average value of 100 for the number of discharges that occur over the earth's surface per second is used, together with a value for the time constant of the cavity, $1 / \Omega_{1}$, of $1 / 16.3$ sec [Rycroft and Wormell, 1964a]. In this time there occur about $100 / 16.3 \simeq 6$ flashes, which tend to excite 
the cavity into resonance, but at random phase angles. Therefore, $\sqrt{6}$ times the $8 \mathrm{~Hz}$ component in the spectrum of one "mean" flash may be inserted for the quantity $I d s(8 \mathrm{~Hz})$ in (16). Thunderstorms tend to occur in the range $\theta=30$ to $140^{\circ}$, so a mean value for $P_{1}(\cos \theta)$ might be $1 / 2$. Using Galejs' [1963] analysis,

$$
\sqrt{\nu(\nu+1)} \simeq\left(\nu+\frac{1}{2}\right)=k R S \simeq k R \operatorname{Re}(\mathrm{S})=1.74,
$$

for $n=1$.

Taking a model ionosphere sharply bounded at $h=76$ $\mathrm{km}$, with $R=6370 \mathrm{~km}, E_{r}$ is found to be approximately $0.2 \mathrm{mV} / \mathrm{m}$ in a $1-\mathrm{Hz}$ bandwidth at $8 \mathrm{~Hz}$, which is in good agreement with the measured value of $0.34 \pm 0.01 \mathrm{mV} / \mathrm{m}$, or $0.26 \mathrm{mV} / \mathrm{m}$ having subtracted the background spectrum shown in figure 6 .

\section{Summary}

In this paper experimentally obtained evidence has been presented which shows that resonant modes of the electromagnetic field in the dielectric cavity between the good conducting earth and ionosphere are observed. At all times when observations of the natural vertical electric field in the atmosphere could be made-at meteorologically calm periods-statistically significant resonances of the fundamental mode at $7.8 \mathrm{~Hz}$ and of the second harmonic at $14.1 \mathrm{~Hz}$ are detected. The next two higher harmonics, at 20.0 and $26.0 \mathrm{~Hz}$, are often apparent. The standard error in these mean resonant frequencies, derived from 31 records, is typically $\pm 0.2 \mathrm{~Hz}$. The mean power in a $1 . \mathrm{Hz}$ bandwidth at $8 \mathrm{~Hz}$ is observed to be 0.117 $\pm 0.008(\mathrm{mV} / \mathrm{m})^{2} / \mathrm{Hz}$. Spectra recorded by Gendrin and Stefant in France show striking similarities with those presented here, whereas those taken in Massachusetts by Balser and Wagner are somewhat different.

These resonances are excited by energy from tropical lightning discharges. Diurnal and seasonal variations of the power in the various modes are interpreted in terms of the movement of thunderstorm centers and nodes of the field. The half width of a model thunderstorm belt, calculated for a model ionosphere with an exponential conductivity versus height profile [Galejs, 1963], is in agreement with thunderstorm statistics. With this model ionosphere the attenuation rate in the band 7 to $30 \mathrm{~Hz}$ is found to be approximately $0.25 \mathrm{~dB} / \mathrm{Mm}$, and the phase velocity $0.77 \mathrm{c}$.

For the longest records taken, the fine structure of the spectrum is investigated and found to be not statistically significant.

Below $5 \mathrm{~Hz}$, the power spectral density of the electric field is relatively immense, and is mainly associated with windborne local space charges or mechanical oscillations of the antenna in the fair-weather electric field.

Michael J. Rycroft expresses his gratitude to T. W. Wormell for introducing him to this exciting field of research, to C. D. Walshaw for aid in constructing the digitizer and for writing computer programs, to the Director of the Mathematical Laboratory for permission to use the EDSAC computer, to the Department of Scientific and Industrial Research for a maintenance grant whilst a research student at Cambridge University, and to the National Academy of SciencesNational Research Council for an Associateship at the NASA Ames Research Center. He also thanks the many people from all parts of the world with whom he has enjoyed stimulating discussions during the course of the work reported here.

\section{References}

Balser, M., and C. A. Wagner (Nov, 19, 1960), Observations of earth-ionosphere resonances, Nature 188, No. 4751, 638-641. Balser, M., and C. A. Wagner (Feb. 1962), Diurnal power variations of the earth-ionosphere cavity modes and their relationship to worldwide thunderstorm activity, J. Geophys. Res. 67, No. 2, 619-625.

Boyer, J. M. (May 13, 1961), A radio wave mechanism to account for the known distribution of Van Allen belts about the earth, Nature 190, No. 4776, 597-599.

Chapman, F. W., and D. Llanwyn Jones (May 16, 1964), Earthionosphere cavity resonances and the propagation of extremely low frequency radio waves, Nature 202, No. 4933, 654-657.

Galejs, J. (Sept. 1961), Terrestrial extremely low frequency noise spectrum in the presence of exponential ionospheric conductivity profiles, J. Geophys. Res. 66, No. 9, 2787-2792.

Galejs, J. (July 1963), Terrestrial extremely-low-frequency propagation, NATO Advanced Study Institute, Low Frequency Electromagnetic Radiation, Bad Homburo, Germany.

('ralejs, J., and R. V. Row (Jan.-Feb. 1964), Propagation of ELF waves below an inhomogeneous anisotropic ionosphere, IEEE Trans. Ant. Prop. AP-12, No. 1, 74-83.

Gendrin, R., and R. Stefant (1964), Magnetic records between 0.2 and 30 cycles per second, Communication presented at AGARD Conference, Propagation of radio waves at frequencies below $300 \mathrm{kc} / \mathrm{s}$, Munich, Germany, Sept. 1962, AGARDograph 74, ed. W. T. Blackband, pp. 371-400. (Pergamon Press, London and New York, N.Y.).

Goldbert, P. A. (Jan. 1, 1963), Effects of cosmic radiation on the extremely low-frequency properties of the mesosphere, J. Geophys. Res. 68, No. 1, 101-110.

Handbook of Geophysics (1960), US Air Force, Cambridge Res. Ctr., Geophys. Res. Directorate, rev. ed. (Macmillan, New York, N.Y.).

Harris, F. B., and R. L. Tanner (July-Aug. 1962), A method for the determination of lower ionosphere properties by means of field measurements on sferics, J. Res. NBS 66D (Radio Prop.), No. 4, 463-478.

Holzer, R. E., and O. E. Deal (Mar. 17, 1956), Low audio-frequency electromagnetic signals of natural origin, Nature 177, No. 4507, 536-537.

Kleimenova, N. (;. (Dec. 1963), Present ideas regarding the nature of high-frequency variations $(1 \mathrm{c} / \mathrm{s}$ to $1 \mathrm{kc} / \mathrm{s})$ in the electromagnetic field of the earth, Bull. Acad. Sci. USSR, Geophys. Ser. No. 12, 1798-1813, A.G.U. Translation, No. 12, 1091-1100.

Lahiri, B. N., and A. T. Price (Jan. 1939), Electromagnetic induction in non-uniform conductors, and the determination of the conductivity of the earth from terrestrial magnetic variations, Phil. Trans. Roy. Soc. London, Ser. A 237, No. 784, 509-540.

Large, M. I., and T. W. Wormell (1958), Fluctuations in the vertical electric field in the frequency range from $1 \mathrm{c} / \mathrm{s}$ to $500 \mathrm{c} / \mathrm{s}$, Recent Advances in Atmospheric Electricity, ed. L. G. Smith, pp. 603607 (Pergamon Press, London and New York, N.Y.).

Llanwyn Jones, D. (Sept. 1963), Observations on extremely lowfrequency (ELF) electromagnetic noise of terrestrial origin, $\mathrm{Ph}$. D. Thesis, London.

Lokken, J. E., J. A. Shand, and C. S. Wright (Feb. 1, 1963), Some characteristics of electromagnetic background signals in the vicinity of one cycle per second, J. Geophys. Res. 68, No. 3, 789-794. 
Madden, T. R., et al. (May 1962), Progress report on geomagnetic studies and electrical conductivity in the earth's crust and upper mantle, Project NR-371-401.

Pierce, E. T. (July-Aug. 1960), Some ELF phenomena, J. Res. NBS $64 D$ (Radio Prop.), No. 4, 383-386.

Pierce, E. T. (July 1, 1963), Excitation of earth-ionosphere cavity resonances by lightning flashes, J. Geophys. Res. 68, No. 13, 4125-4127.

Row, R. V. (Nov.-Dec. 1962), On the electromagnetic resonant frequencies of the earth-ionosphere cavity, IRE Trans. Ant. Prop. AP-10, No. 6, 766-769.

Rycroft, M. J., and T. W. Wormell (1964a), The natural ELF electromagnetic noise in the band $2-40 \mathrm{c} / \mathrm{s}$; apparatus and some preliminary results, Communication presented at AGARD Conference, Propagation of radio waves at frequencies below $300 \mathrm{kc} / \mathrm{s}$, Munich, Germany, Sept. 1962, AGARDograph 74, ed. W. T. Blackband, pp. 421-434 (Pergamon Press, London and New York, N.Y.).

Rycroft, M. J., and T. W. Wormell (1964b), The extremely low frequency spectrum of a "mean" lightning discharge (private communication).

Schumann, W. O. (Feb. 1952), Über die strahlunglosen eigenschwingungen einer leitenden Kugel, die von einer Luftschicht und einer Ionosphärenhulle ungeben ist, Z. Naturforschg. 7a, Heft 2, 149-154.

Stefant, R. (July-Sept. 1963), Application d'un magnetomètre à l'induction à la détection des fréquences de résonance de la cavité terre-ionosphère, Ann. Geophys. 19, No. 3, 250-283.

Swinnerton-Dyer, H. P. F. (Jan. 1963), The calculation of power spectra, Computer J. 5, No. 1, 16-23.

Thompson, W. B. (June 1963), A layered model approach to the earth-ionosphere cavity resonance problem, Ph. D. Thesis, MIT.
Williams, J. C. (June 1959), Thunderstorms and VLF radio noise, $\mathrm{Ph}$. D. Thesis, Harvard.

Wait, J. R. (Mar-Apr. 1960), Terrestrial propagation of verylow-frequency radio waves: a theoretical investigation, J. Res. NBS 64D (Radio Prop.), No. 2, 153-204.

Wait, J. R. (1962), Electromagnetic Waves in Stratified Media, Chapter X (Pergamon Press, London and New York, N.Y.).

Wait, J. R. (Apr. 1964), On the theory of Schumann resonances in the earth-ionosphere cavity, Can. J. Phys. 42, No. 4, 575-582.

Watt, A. D., F. S. Mathews, and E. L. Maxwell (June 1963), Some electrical characteristics of the earth's crust, Proc. IEEE 51, No. 6, 897-910.

Zukerman, L. G. (June 1961), Application of a spectrum analyzer for use with random functions, IRE Trans. Instr. 1-10, No. 1, 37-43.

\section{Additional References}

Chapman, C. W. (Oct. 1962), A solution for ELF propagation in the earth-ionosphere cavity bounded by an inhomogeneous, anisotropic ionospheric medium, Report No. 6-50, AFCRL-62-792.

Rycroft, M. J. (Oct. 1964), Low frequency distrubances of natural origin of the electric and magnetic fields of the earth, Ph. D. Thesis, Cambridge.

(Paper 69D8-540) 